\title{
An alternative route to detect parity violating energy differences through Bose-Einstein condensation of chiral molecules
}

\author{
Pedro Bargueño, ${ }^{* a b}$ Ricardo Pérez de Tudela, ${ }^{a}$ Salvador Miret-Artés ${ }^{a}$ and \\ Isabel Gonzalo ${ }^{c}$
}

Received 17th June 2010, Accepted 18th August 2010

DOI: $10.1039 / \mathbf{c 0 c p 0 0 9 0 7 e}$

Interactions which do not conserve parity might influence chiral compounds giving rise to a parity violating energy difference (PVED) that might have affected the evolution towards homochirality. However, this tiny effect predicted by electroweak-quantum chemistry calculations is easily masked by thermal effects, making it desirable to reach cold regimes in the laboratory. As an alternative route to the detection of the PVED, we study a simplified model of Bose-Einstein condensation of a sample of non-interacting chiral molecules, showing that it leads to a nonzero optical activity of the condensate and also to a subcritical temperature in the heat capacity, due to the internal structure of the molecule characterized by tunneling and parity violation.

This predicted singular behavior found for the specific heat, below the condensation temperature, might shed some light on the existence of the thus far elusive PVED between enantiomers.

\section{Introduction}

Since the prediction and subsequent discovery of parity violation $^{1,2}$ in weak interactions, the role of discrete symmetry breaking in physics and chemistry is an intriguing field of research. Although the weak interaction (between electrons and nuclei) mediated by the gauge $Z^{0}$ boson has been extensively studied and observed in atoms, ${ }^{3}$ it has only been predicted in molecules. In molecular systems, the theory of electroweak interactions predicts a parity violating energy difference (PVED) between the two enantiomers of chiral molecules to be between $10^{-13}$ and $10^{-21} \mathrm{eV}$, including polypeptides, RNA and DNA molecules. ${ }^{4-14}$ However, no conclusive energy difference has been reported, for example, in experimental spectroscopic studies of the $\mathrm{CHBrClF}$ molecule reaching an energy resolution of about $10^{-15} \mathrm{eV} .^{15,16}$

The importance of this symmetry-breaking in molecules is twofold: (i) at a fundamental level, the intrinsic chiral nature which is present in some molecules should reflect the underlying interaction containing pseudoscalar magnitudes such as those appearing in the weak interaction; and (ii) it could be intimately related to the origin of homochirality, that is, the almost exclusive one-handedness of the chiral molecules found in living systems, e.g. L-amino acids and D-sugars, this being one of the most fascinating open problems which links fundamental physics with the biochemistry of life. ${ }^{17}$ We note that, although the PVED has been proposed to tip the balance away from racemic mixtures toward an enhancement that could be incorporated into primitive forms of life, this energy difference is extremely small, so that amplification of some sort would be required to produce a detectable effect at a

\footnotetext{
${ }^{a}$ Instituto de Física Fundamental (CSIC), Serrano 123,

28006 Madrid, Spain.E-mail: pbdr@iff.csic.es

${ }^{b}$ Departamento de Química Física, Universidad de Salamanca, 37008 Salamanca, Spain

${ }^{c}$ Departamento de Óptica, Universidad Complutense de Madrid, 28040 Madrid, Spain
}

macroscopic level. However, in a recent work, MacDermott and coworkers ${ }^{18}$ conclude that parity-violation does indeed favour the neutral $\mathrm{L}$ form of the $\alpha$-methyl amino acids found to show L-excesses in the Murchison meteorite.

But although the PVED could play some role in the evolution to homochirality, there is a problem concerning its detection due to the fact that the tiny parity violating signals are easily masked by thermal effects, thus making it highly desirable to reach cold or ultracold regimes in the laboratory. ${ }^{19-22}$ The measurement of fundamental physical properties such as the PVED between enantiomers could be achieved by trapping molecules at low temperatures (in the milliKelvin range or below) and, subsequently, performing ultrahigh-resolution spectroscopic measurements of vibrational or electronic transitions. We would like to point out that these temperatures, and even colder, have been reached within the actual experimental capabilities only for atoms and diatomic molecules. The rapid expansion of the research field of ultracold chemistry is already opening new and exciting possibilities concerning more complex systems. ${ }^{23}$ Thus, the study of quantum thermal effects in chiral molecules through pseudoscalar operators as the basic object describing their thermodynamics is of fundamental importance. Very recently, we have studied the corresponding classical thermodynamics of non-interacting chiral molecules. ${ }^{24,25}$ In this sense, as pointed out by Flambaum and coworkers concerning some capabilities of Bose-Einstein condensates to amplify weak interactions in chiral molecules, ${ }^{19}$ the study of Bose-Einstein condensation (BEC) for a gas of chiral molecules could shed some light on some properties relevant to determine the thus far elusive PVED.

Concerning this Bose-Einstein amplification of weak interactions, Salam proposed that the existence of the PVED, together with a type of BEC phenomenon, may lead to a second-order phase transition below a critical temperature allowing D-aminoacids (the less stable) to tunnel into the more stable one. ${ }^{26,27}$ This temperature was identified with that of the 
Bardeen, Cooper and Schrieffer theory of superconductivity and was supposed to lie close to $250 \mathrm{~K}$. Although recent extensive experimental studies have been devoted to test Salam's hypothesis, ${ }^{28}$ it is generally accepted that this phase transition is kinetically hindered and a Bose-Einstein condensation of this kind is not expected to take place in the solid state. ${ }^{29}$ However, the capability of BEC to amplify quantum effects to the macroscopic scale could be useful to detect the PVED, at least in the gas phase.

In this sense, for a Bose gas of chiral molecules, the inclusion of extra information about the internal structure of such molecules could determine some macroscopic properties relevant to obtain the PVED such as, for example, the optical activity and heat capacity (the study of cold atoms with intrinsic spin structure is currently a very hot topic, including two-component condensates, spinor condensates, etc. ${ }^{30}$ ). To this end, we will employ a two-state model which includes tunneling and parity violating effects in chiral molecules (this approach was first considered in ref. 31 and is widely used to describe, for instance, the internal dynamics of chiral molecules). Although the two-state (or spin 1/2) model is well known in many fields, including spin tunneling, magnetization, and quantum dissipative systems, to name only a few, this is the first time, to the best of our knowledge, that this model has been employed to study BEC of chiral molecules. Following the seminal works on $\mathrm{BEC}$, predicted for a non-interacting gas and carried out in numerous dilute atomic gases ${ }^{32}$ and in some diatomic molecules, such as ${ }^{40} \mathrm{~K}_{2}$, ${ }^{33}$ we will assume that the interaction among molecules is negligible to first order and, therefore, a non-interacting quantum gas of chiral molecules will be considered. We point out that although we are aware of the importance of interparticle interactions to study the thermodynamics at ultracold temperatures, our approach of non-interacting bosons constitutes the zeroth-order approach and retains the essential characteristics of the imprint of chirality in BEC. The very low densities needed to achieve $\mathrm{BEC}$ with chiral molecules tend to reduce the interaction among them.

In this article we show that a condensed gas of noninteracting chiral molecules displays optical activity related to the PVED. In addition, we show a dramatic change of the heat capacity with temperature, leading to the appearance of a secondary maximum (developing a shoulder-type-structure) associated with the Schottky anomaly below the condensation temperature. This subcritical temperature is expected to lie in the cold or ultracold regime.

In an ideal Bose-Einstein gas, only the translational part of the molecular motion is involved and the critical temperature for $\mathrm{BEC}$ is reached when the fugacity of the gas $z=\exp (\beta \mu)$, with $\mu$ being the chemical potential and $\beta=\left(k_{\mathrm{B}} T\right)^{-1}$, is approaching unity (assuming that the energy of the ground state is zero). ${ }^{34}$ This $T_{\mathrm{c}}$ depends on the molecule mass and the density of the system. At $T<T_{\mathrm{c}}$, there must be a nonnegligible fraction of molecules in the ground state, the internal energy goes with $T^{5 / 2}$ and the heat capacity with $T^{3 / 2}$. If each chiral molecule in the condensed phase has its own internal structure, we could ask ourselves if the corresponding quantum statistics describing the thermodynamic behavior, intimately related to the PVED, can be manifested by a macroscopic effect. For this goal, a two-state model is assumed for each chiral molecule.

\section{Thermodynamics of the two-state model: a brief summary}

In the absence of parity violation, the dynamics of a chiral molecule is well described by a symmetric double well potential. In this case, the true stationary states of a chiral molecule are the (achiral) eigenstates of parity. The effect of including in the Hamiltonian the internal P-odd term given by the parity violating electronnucleon electroweak interaction, $H^{\mathrm{PV}}$, introduces an energy difference between the two minima of the double well potential, leading to a new set of energy eigenstates. Let us consider the total Hamiltonian of the system $H=H^{0}+H^{\mathrm{PV}}$, with $H^{0}$ including only parity conserving terms. Using a two-state model, we choose the left and right chiral states bases, $|L\rangle$ and $|R\rangle$ (localized respectively in the left and right minimum of the double well potential), in order to show clearly the parity properties of the Hamiltonian: ${ }^{31}$

$$
H=H^{0}+H^{\mathrm{PV}}=\delta \sigma_{x}+\varepsilon_{\mathrm{PV}} \sigma_{z}
$$

where $\sigma_{x, z}$ are the Pauli matrices. The eigenstates of $H,|1,2\rangle$, can be expressed as linear combinations of the chiral states by means of a rotation where the corresponding mixing angle, $\theta$, obtained from a knowledge of the eigenvectors of $H$, is given by tan $2 \theta=2 H_{L R} /\left(H_{R R}-H_{L L}\right)=\left(\delta / \varepsilon_{\mathrm{PV}}\right)$. The energy splitting between the two eigenstates of $H^{0}$ is $2 \delta>0$, where $\delta=$ $\left\langle L\left|H^{0}\right| R\right\rangle$. This magnitude is related to the height of the barrier of the double well potential and is inversely proportional to the tunneling time. The PVED is then given by $\left|H_{R R}-H_{L L}\right|=$ $\left|2 \varepsilon_{P V}\right|$ with $\varepsilon_{P V}=\left\langle R\left|H^{\mathrm{PV}}\right| R\right\rangle=-\left\langle L\left|H^{\mathrm{PV}}\right| L\right\rangle$ (we remark that $\varepsilon_{P V}$ is the eigenvalue of a pseudoscalar operator in the chiral basis). The eigenvalues of the system are given by $E_{1,2}=E_{0} \mp \Delta$, with $E_{0}=\left(H_{R R}+H_{L L}\right) / 2$ and $\Delta \equiv \sqrt{\varepsilon_{\mathrm{PV}}^{2}+\delta^{2}}$ (hereafter we will take $E_{0}=0$ for the sake of simplicity).

\section{Heat capacity contribution of the internal structure}

In a gas of non-interacting chiral molecules at temperature $T$, the inclusion of P-odd effects leads one to deal with a pseudoscalar operator for obtaining any thermodynamic variable. $^{24,25}$ Thus, for a system described by a biased double well potential, the only magnitude which, roughly speaking, distinguishes between left and right conformations is the population difference between both wells, $N_{L}-N_{R}$, which is directly related to the optical activity of the system. If $X$ is a pseudoscalar operator, it can be shown that

$$
\left|\langle X\rangle_{\text {stat }}\right|=|x|\left(N_{L}-N_{R}\right)_{\text {stat }},
$$

where $\pm x$ are the eigenvalues of $X$ and the subscript stat stands for the different statistics considered. Eqn (2) is a generalization to any statistics of the Maxwell-Boltzmann (MB) thermal average. ${ }^{24}$ If $N_{1,2}$ is the number of particles in the ground, $|1\rangle$, and the excited state, $|2\rangle$, respectively, 
a straightforward calculation gives the expression for $N_{2}-N_{1}$ to be

$$
\left(N_{2}-N_{1}\right)_{\text {stat }}=\tanh \beta \Delta\left(1+p \frac{z+z^{-1}}{2} \operatorname{sech} \beta \Delta\right)^{-1}
$$

where $p=0$ applies for MB, $p=-1$ for Bose-Einstein (BE) and $p=+1$ for Fermi-Dirac statistics. We remark that, when $\varepsilon_{\mathrm{PV}}$ dominates over $\delta$, the true eigenstates of the system are the chiral states, $|L\rangle$ and $|R\rangle$, and changing the basis from $|1\rangle,|2\rangle$ to $|L\rangle,|R\rangle$ introduces the factor $\cos 2 \theta=\varepsilon_{\mathrm{PV}} / \Delta$ leading to

$$
N_{L}-N_{R}=\varepsilon_{\mathrm{PV}}\left(N_{2}-N_{1}\right) / \Delta .
$$

Moreover, $N_{L}-N_{R}$ changes its sign when considering a parity-transformed double well since $\varepsilon_{\mathrm{PV}}$ changes to $-\varepsilon_{\mathrm{PV}}$. From the knowledge of $N_{2}-N_{1}$, the internal energy of the gas is

$$
U=-\Delta\left(N_{1}-N_{2}\right)_{\text {stat }} .
$$

From eqn (5) one can inmediately obtain the heat capacity at constant volume. This $C_{\mathrm{v}}$ can be interpreted as a measure of the fluctuations of the optical activity of the system, that is, a measure of the fluctuations of the pseudoscalar character of the system.

\section{Bose-Einstein gas of chiral molecules}

Let us now consider BE statistics $(p=-1)$ for a gas of two-state chiral molecules, that is, the Hamiltonian $H$ includes tunneling and parity violation. The total number of particles is $N=N_{1}+N_{2}$. Using the standard expression for the occupation numbers for Bose statistics, we get $N=N_{1}+$ $\left(z^{-1} \exp (\beta \Delta)-1\right)^{-1}$. For our purposes, let us define a temperature $T^{*}$ in which $N_{1}=0$ (all the particles are in the excited state $E_{2}$ or, in other words, the number of particles in the ground state is negligible). This definition of the condensation temperature was also pointed out in ref. 35 . This leads to the condensation temperature to be determined from $T^{*}=\frac{2 \Delta}{k_{\mathrm{B}} \ln \left(1+\frac{1}{N}\right)}$.

For $T<T^{*}$ it is easy to see that the gas displays a non-zero optical activity which is proportional to

$$
N_{1}-N_{2}=N\left(1-2 \frac{\exp \left(2 \beta^{*} \Delta\right)-1}{\exp (2 \beta \Delta)-1}\right) .
$$

This enables us to write:

$$
\frac{N_{1}}{N}=1-\frac{\exp \left(2 \beta^{*} \Delta\right)-1}{\exp (2 \beta \Delta)-1},
$$

the average internal energy as

$$
u=\frac{U}{N}=\Delta\left(2 \frac{\exp \left(2 \beta^{*} \Delta\right)-1}{\exp (2 \beta \Delta)-1}-1\right)
$$

and the heat capacity as

$$
C_{v}^{\text {int }}=\frac{\partial u}{\partial T}=4 k_{\mathrm{B}}(\beta \Delta)^{2} \frac{\exp \left(2 \beta^{*} \Delta\right)}{\exp (2 \beta \Delta)-1} .
$$

It is worth noting that this heat capacity reaches its maximum value at the subcritical temperature, $T_{\mathrm{sc}}$,

$$
\beta_{\mathrm{sc}} \Delta \simeq 0.797
$$

which reflects nothing but the Schottky anomaly due to the saturation of the energy levels of the system. Thus, at $T_{\mathrm{sc}}$, the heat capacity shows the splitting of the two levels.

\section{Bose-Einstein condensation of a kinetic gas of two-state chiral molecules}

If we include a kinetic term in the Hamiltonian, the total partition function for an ideal Bose gas of chiral molecules in the two-state model can be written as

$$
Z_{\text {tot }}=Z_{\text {kin }} \cdot Z_{\text {int }}
$$

where the subscripts denote the kinetic and internal contributions, respectively. The first factor can be found in any standard textbook of statistical mechanics ${ }^{34}$ and the second factor is given, for our case, by

$$
Z_{\text {int }}=\prod_{i=1,2}\left(1-z e^{-\beta E_{i}}\right)^{-1}
$$

The factorization of the partition function leads to a sum of the corresponding heat capacities, $C_{v}^{\mathrm{tot}}=C_{v}^{\mathrm{kin}}+C_{v}^{\mathrm{int}}$. We note that there are now two temperature regimes involved in the chiral system: (i) the temperature at which the gas undergoes BEC $\left(T_{\mathrm{c}}\right)$ and (ii) the subcritical temperature $\left(T_{\mathrm{sc}}\right.$, below $\left.T_{\mathrm{c}}\right)$ where the heat capacity displays a maximum. In the case of a free gas, $T_{\mathrm{c}}$ is related to the mass and number density of the bosons but $T_{\mathrm{sc}}$ depends only (for the two-state model) on the energy splitting between the $|1\rangle$ and $|2\rangle$ states. Thus, for $T<T_{\mathrm{c}}, C_{v}^{\mathrm{int}}$ is given by eqn (9) and, for $T>T_{\mathrm{c}}$, the corresponding expression can be obtained from eqn (5). $C_{v}^{\mathrm{kin}}$ is the usual heat capacity for an ideal Bose gas. It is worth pointing out that the very interesting case where $T_{\mathrm{c}}$ and $T_{\mathrm{sc}}$ are of the same order gives rise to an appreciable change in the heat capacity, with a shoulder-type structure below $T_{\mathrm{c}}$. This behavior is displayed in Fig. 1, where the total heat capacity is plotted in terms of the reduced temperature $k_{\mathrm{B}} T / \Delta$ for the cases $T_{\mathrm{sc}}=0.1 T_{\mathrm{c}}$ (top panel) and $T_{\mathrm{sc}}=0.5 T_{\mathrm{c}}$ (bottom panel) (we have assumed $T^{*}=T_{\mathrm{c}}$ ). Strictly speaking, the effect of $\Delta$ on the condensation temperature, $T_{\mathrm{c}}$, results in a very small shift of $T_{\mathrm{c}}$. However, this difference is so tiny that for most practical purposes, we keep $T_{\mathrm{c}}$ instead of the shifted $T_{\mathrm{c}}$. When $T_{\mathrm{c}}=T_{\mathrm{sc}}$, the subcritical temperature corresponds to a very precise density of bosons of mass $m, n_{\mathrm{sc}}$, given by

$$
n_{\mathrm{sc}} \simeq 0.178\left(\frac{m \Delta}{\hbar^{2}}\right)^{3 / 2}
$$

The bottom panel of Fig. 1 should be observed, for example, for the $\mathrm{T}_{2} \mathrm{Se}_{2}$ system. This system has $\varepsilon_{\mathrm{PV}} \approx 2.5 \times 10^{-14} \mathrm{eV}$, $\delta \approx 5 \times 10^{-17} \mathrm{eV}$ and the tunneling time is $\sim 40 \mathrm{~s}$ (see Table 2 of ref. 13). Its subcritical density, $n_{\mathrm{sc}}$, is about $10^{10} \mathrm{~cm}^{-3}$, which is of the order of the critical densities. Thus, in this case, the corresponding discontinuity in the heat capacity could provide us a direct and clear signal of molecular parity violation. 

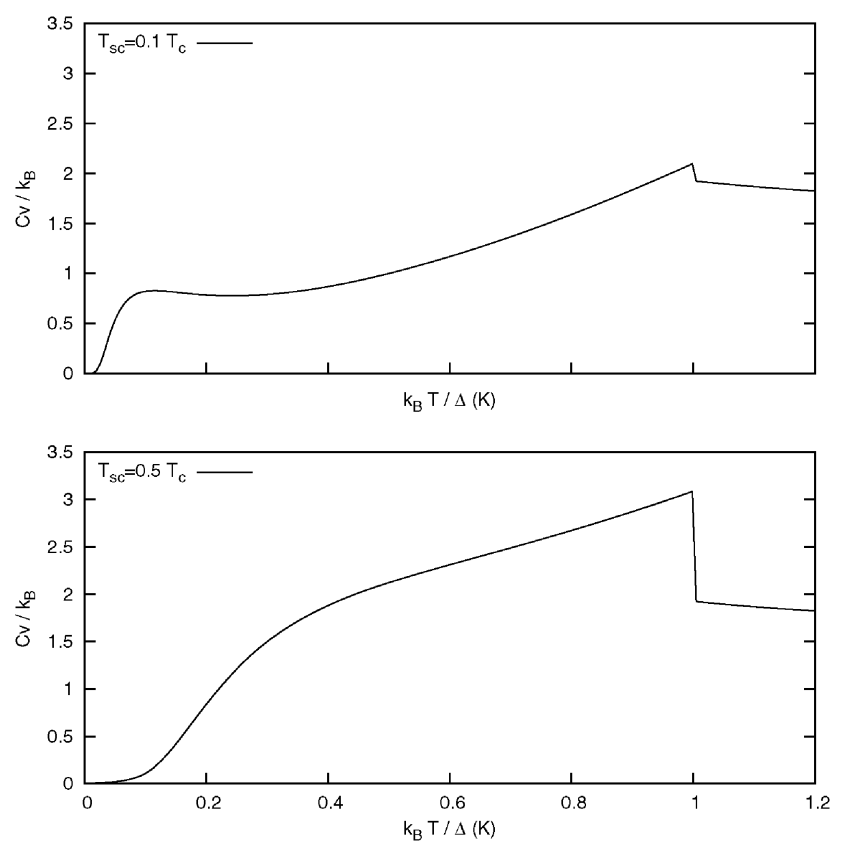

Fig. 1 Total heat capacities for a gas of chiral molecules as a function of the reduced temperature for two cases: $T_{\mathrm{sc}}=0.1 T_{\mathrm{c}}$ and $T_{\mathrm{sc}}=0.5 T_{\mathrm{c}}$. The gas undergoes BEC at $k_{\mathrm{B}} T / \Delta=1$. We have taken $T^{*}=T_{\mathrm{c}}$. See text for symbols.

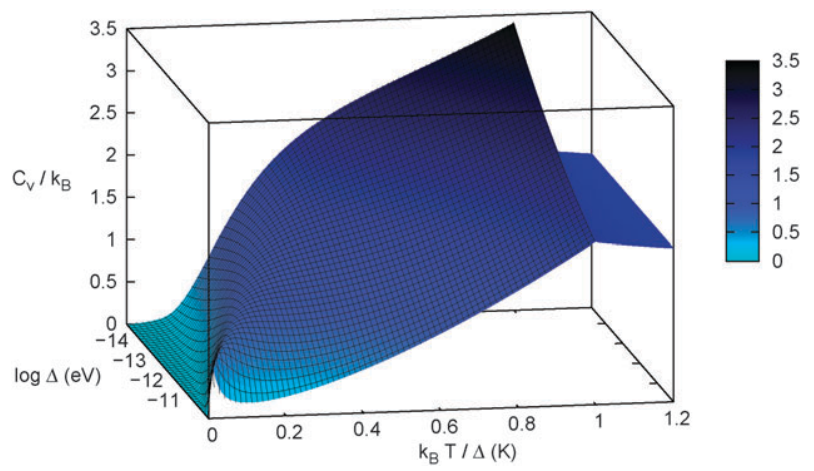

Fig. 2 Three dimensional plot of the heat capacity versus $\Delta$ and the reduced temperature. The gas undergoes BEC at $k_{\mathrm{B}} T / \Delta=1$. We have taken $T^{*}=T_{\mathrm{c}}$. See text for symbols.

In Fig. 2, a three dimensional plot of the heat capacity in the range of variation [0,1.2] of the reduced temperature and $\left[10^{-15}, 10^{-10}\right]$ of the splitting $\Delta(\mathrm{eV})$ is shown. This plot clearly shows the overall shoulder-type structure as well as the subcritical maximum for different chiral molecules, characterized by the splitting $\Delta$. This splitting is representative of molecules such as, for example, $\mathrm{H}_{2} \mathrm{~S}_{2}, \mathrm{CHBrClF}$ and $\mathrm{H}_{2} \mathrm{Se}_{2} .{ }^{13}$

\section{Discussion and conclusions}

The subcritical maximum (or anomalous shoulder-type structure) found here could be considered as a signal of the Schottky anomaly which comes from lifting the degeneracy of the internal degrees of freedom. Although this was previously considered in the framework of a path integral approach to study BEC in spinor gases, ${ }^{36}$ our simple model allows us to identify the anomaly more clearly. We would like to stress that the subcritical temperature is determined in essence by $\delta$ and $\varepsilon_{\mathrm{PV}}$, these temperatures lying in the cold or ultracold regime. The existence of such temperatures could be possible for any system whose constituents have internal ground states slightly split, such as for example: the inversion doubling of non-rigid pyramidal molecules, the splitting due to torsional or internal rotations through potential barriers in non-rigid molecules, or eventually the hyperfine structure of the ground state of atoms and molecules (in this last case, it could be more easy to verify the type of anomaly here studied). However, chiral molecules in this two-state model play a special role in BEC when the splitting is mainly due to parity violation (as in $\mathrm{T}_{2} \mathrm{Se}_{2}$ ). In this case, an observation of the anomaly predicted in the heat capacity provides direct information about the PVED. In addition, when $\varepsilon_{\mathrm{PV}}$ determines the dynamics, then the eigenstates of the system tend to be the chiral states $|L, R\rangle$. We also point out that a measurement of the optical activity of the condensate would be a confirmation of the existence of the PVED, noting that the factor $\varepsilon_{\mathrm{PV}} / \Delta$, which is the optical activity for zero temperature, should be measurable by state-of-the-art of actual polarimeters, as we concluded in ref. 25.

Finally, we would like to point out that the energy scale of molecular parity violation is associated with the natural scale of densities which corresponds to those achieved in Bose-Einstein condensates. Thus, BEC seems to be an alternative route to detect the PVED. Furthermore, as is well known, interactions could modify the physics of the problem, in particular by shifting the condensation temperature, so it is fundamental to ask ourselves to what extent the anomaly in the heat capacity persists when we consider an interacting system of chiral molecules. Although one could introduce the interaction between molecules under the Gross-Pitaevskii or any other more sophisticated treatment, an alternative way is to study the molecular sample as an open quantum system under the influence of dissipation. In this sense, we note that the heat capacity anomalies of open quantum systems have been recently studied when coupled to a thermal bath, ${ }^{37}$ showing the robustness of these anomalies when the interaction is taken into account. Thus, we expect that a similar stability for the anomaly here considered will persist even in presence of an environment. The study of heat capacity anomalies in BEC chiral gases embedded in appropriately chosen environments is currently in progress.

Concerning biological chiroselection, all proposed mechanisms of discrimination between the two enantiomers, even those producing very small effects, like parity violation, are usually taken in account since several amplification processes may be present along many years. Thus, under the influence of appropriate amplification mechanisms, parity violation can not be ruled out as a possible origin of homochirality. In an attempt to provide experimental basis of the existence of this symmetry breaking effect, we have explored an alternative way to detect it through Bose-Einstein condensation.

This work has been funded by the MEC (Spain) under projects CTQ2008-02578/BQU, FIS2007-62006 and FIS2007-65382, supported by grants BES-2006-11976 (P. B.) and BES-2006-7454 (R. P. de T.). P. B. dedicates this work to Anaís Dorta-Urra for her help and encouragement during recent months. 


\section{References}

1 T. D. Lee and C. N. Yang, Phys. Rev., 1956, 104, 254.

2 C. S. Wu, E. Ambler, R. W. Hayward, D. D. Hoppes and R. P. Hudson, Phys. Rev., 1957, 105, 1413.

3 A. M. Bouchiat and C. C. Bouchiat, Rep. Prog. Phys., 1997, 60, 1351.

4 R. Zanasi, P. Lazzeretti, A. Ligabue and A. Soncini, Phys. Rev. E: Stat. Phys., Plasmas, Fluids, Relat. Interdiscip. Top., 1999, 59, 3382.

5 J. K. Laerdahl, P. Schwerdtfeger and H. M. Quiney, Phys. Rev. Lett., 2000, 84, 3811.

6 J. Thyssen, J. K. Laerdahl and P. Schwerdtfeger, Phys. Rev. Lett., 2000, 85, 3105.

7 A. S. Lahamer, S. M. Mahurin, R. N. Compton, D. House, J. K. Laerdahl, M. Lein and P. Schwerdtfeger, Phys. Rev. Lett., 2000, 85, 4470 .

8 F. Faglioni, P. S. D'Agostino, B. Cadioli and P. Lazzeretti, Chem. Phys. Lett., 2005, 407, 522.

9 F. Faglioni, A. Passalacqua and P. Lazzeretti, Origins Life Evol. Biosphere, 2005, 35, 461.

10 F. Faglioni, I. G. Cuesta and P. Lazzeretti, Chem. Phys. Lett., 2006, 432, 263.

11 P. Soulard, P. Asselin, A. Cuisset, J. R. Aviles Moreno, T. R. Huet, D. Petitprez, J. Demaison, T. B. Freedman, X. Cao, L. A. Nafie and J. Crassous, Phys. Chem. Chem. Phys., 2006, 8, 79.

12 D. Figgen and P. Schwerdtfeger, Phys. Rev. A: At., Mol., Opt. Phys., 2008, 78, 012511.

13 M. Quack, J. Stohner and M. Willeke, Annu. Rev. Phys. Chem., 2008, 59, 741.

14 D. Figgen, A. Koers and P. Schwerdtfeger, Angew. Chem. Int. Ed., 2010, 49, 2941.

15 C. Daussy, T. Marrel, A. Amy-Klein, C. T. Nguyen, C. J. Bordé and C. Chardonnet, Phys. Rev. Lett., 1999, 83, 1554.

16 J. Crassous, C. Chardonnet, T. Saue and P. Schwerdtfeger, Org. Biomol. Chem., 2005, 3, 2218.

17 A. Guijarro and M. Yus, The Origin of Chirality in the Molecules of Life, RSC Publishing, Cambridge, 2009.
18 A. J. MacDermott, T. Fu, R. Nakatsuka, A. P. Coleman and G. O. Hyde, Origins Life Evol. Biosphere, 2009, 39, 459.

19 V. V. Flambaum and J. S. M. Ginges, Phys. Rev. A: At., Mol., Opt. Phys., 2006, 74, 025601.

20 P. Blythe, B. Roth, U. Frohlich, H. Wenz and S. Schiller, Phys. Rev. Lett., 2005, 95, 183002.

21 J. M. Hudson and P. Soldan, Int. Rev. Phys. Chem., 2006, 25, 497.

22 J. M. Hudson and P. Soldan, Int. Rev. Phys. Chem., 2007, 26, 1.

23 L. D. Carr and J. Ye, New J. Phys., 2009, 11, 055049.

24 P. Bargueño, I. Gonzalo, R. Pérez de Tudela and S. Miret-Artés, Chem. Phys. Lett., 2009, 483, 204.

25 I. Gonzalo, P. Bargueño, R. Pérez de Tudela and S. Miret-Artés, Chem. Phys. Lett., 2010, 489, 127.

26 A. Salam, J. Mol. Evol., 1991, 33, 105.

27 A. Salam, Phys. Lett. B, 1992, 288, 153.

28 W. Wang, F. Yi, Z. Zhao, X. Jin and Y. Tang, J. Biol. Phys., 2000, 26, 51 .

29 R. Sullivan, M. Pyda, J. Pak, B. Wunderlich, J. R. Thompson, R. Pagni, H. Pan, C. Barnes, P. Schwerdtfeger and R. Compton, J. Phys. Chem. A, 2003, 107, 6674.

30 C. J. Pethick and H. Smith, Bose-Einstein Condensation in Dilute Gases, Cambridge University Press, 2002.

31 R. A. Harris and L. Stodolsky, Phys. Lett. B, 1978, 78, 313.

32 M. H. Anderson, J. R. Ensher, M. R. Matthews, C. E. Wieman and E. A. Cornell, Science, 1995, 269, 198.

33 C. A. Regal, C. Ticknor, J. L. Bohn and D. S. Jin, Nature, 2003, 424, 47.

34 R. P. Feynman, Statistical Mechanics, Advance Books Classics, Addison-Wesley, 1998.

35 R. K. Pathria, Statistical Mechanics, Pergamon Press, Oxford, 1972.

36 L. F. Lemmens, F. Brosens and J. T. Devreese, Phys. Rev. E: Stat. Phys., Plasmas, Fluids, Relat. Interdiscip. Top., 2000, 61, 3358.

37 G.-L. Ingold, P. Hänggi and P. Talkner, Phys. Rev. E: Stat., Nonlinear, Soft Matter Phys., 2009, 79, 061105. 